\title{
Organism-induced habitat restoration leads to bi-stability in metapopulations
}

\author{
Feng Zhang ${ }^{\mathrm{a}, \mathrm{b}, *}$, Yi Tao ${ }^{\mathrm{c}}$, Cang Hui ${ }^{\mathrm{a}}$ \\ ${ }^{a}$ Centre for Invasion Biology, Department of Botany and Zoology, Stellenbosch University, Private Bag X1, Matieland 7602, South Africa \\ ${ }^{\mathrm{b}}$ Department of Mathematics, College of Science, Gansu Agricultural University, Lanzhou 730070, China \\ ${ }^{\mathrm{c}}$ Key Laboratory of Animal Ecology and Conservation Biology, Institute of Zoology, Chinese Academy of Science, Beijing 100101, China
}

\section{A R T I C L E I N F O}

\section{Article history:}

Received 9 March 2012

Received in revised form 23 August 2012

Accepted 31 August 2012

Available online 13 September 2012

\section{Keywords:}

Habitat destruction

Habitat restoration

Threshold phenomenon

\begin{abstract}
A B S T R A C T
Following Levins' patch occupancy model, we presented a differential-equation model, in which both the metapopulation dynamics and the dynamics of the fraction of suitable patches in the habitat are characterized. Habitat restoration induced by organism itself (internal restoration) and by other organisms or/ and abiotic causes (external restoration) were incorporated in the model, together with habitat destruction. Stability analysis revealed the existence of alternative equilibriums (i.e., bi-stability) in the system. The internal restoration of habitat was identified as the trigger for the bi-stability, whereas the external restoration, in contrast, can eliminate the bi-stability from the system. The results, thus, emphasize the important role of the organism-environment feedback in biological conservation.
\end{abstract}

(c) 2012 Elsevier Inc. All rights reserved.

\section{Introduction}

Habitat destruction and fragmentation is the prime process causing current mass biodiversity loss [1]. As a result, metapopulation theories have been widely applied in conservation and spatial ecology [2]. The classic metapopulation model is developed by Levins $[3,4]$ and has contributed critical insights into the conservation of species on fragmented landscapes [2-4]. Levins' patch occupancy model can be further modified by incorporating habitat destruction [5-9]. Such a modification has led to the Levins rule in conservation biology, estimating the minimum amount of suitable habitat necessary for the long-term persistence of metapopulations based only on the colonization and extinction rates of focal species.

The patch occupancy model, however, assumes that the fraction of suitable habitat remains a constant in the landscape, which is often not the case in real systems. In reality, the size of suitable habitat is constantly fluctuating due to the habitat destruction and restoration. As an alternative to human restoration, organisms themselves could also induce habitat restoration. All organisms affect their environments on various temporal and spatial scales $[10,11]$. The modification and creation of environment by organisms could potentially facilitate habitat restoration and thus alter the available amount of suitable habitat to themselves or/and other species [12]. For example, soil microorganisms can form biological soil crusts and protect soil surface from erosion, preserving

* Corresponding author at: Centre for Invasion Biology, Department of Botany and Zoology, Stellenbosch University, Private Bag X1, Matieland 7602, South Africa. Tel.: +27 21808 3413; fax: +27218082995.

E-mail address: fzhang@sun.ac.za (F. Zhang). nitrogen and carbon to the soil [13]. Ants, termites and earthworms can also shift the soil profile towards their favorite conditions [10]. Dune plants can reinforce and stabilize their habitat on drift sand [14]; desert plants can accumulate soil content around them and facilitate their future recruitment [15]. These organism-environment feedbacks can cause complex spatial patterns of species distributions [16-19]. Therefore, when evaluating metapopulation persistence, we should include not only habitat destruction (or degradation) and human restoration in the population dynamics, but also the fine-scale organism-induced habitat restoration.

Following Levins' $[3,4]$ patch occupancy model, we presented a differential-equation model that describes the dynamics not only of metapopulations but also of suitable habitat over time. The model distinguished the internal habitat restoration induced by focal species itself from the external restoration by other species or abiotic causes. The equilibriums and stability of the system were analyzed mathematically. Our main goal is to reveal the relationship between suitable habitat size (affected by habitat destruction, internal and external restoration processes) and metapopulation persistence, and further investigate the ecological implication of this relationship. Our model and results thus emphasize the effect of organism-environment feedbacks on the system stability and dynamical complexity.

\section{Model}

Metapopulations describe a group of local populations inhabiting a patch network linked by migration between patches. The metapopulation theory explains that, even if local populations are 
subject to extinction, the long-term persistence of the species can still be achieved at regional level [2]. The classical metapopulation framework is based on the Levins $[3,4]$ patch occupancy model:

$\frac{d p}{d t}=c p(h-p)-e p$,

where $p$ is the fraction of patches occupied by the species (or called the metapopulation size); parameter $c$ and $e$ are the colonization and extinction rates, respectively; parameter $h$ denotes the fraction of suitable patches (thus $1-h$ indicates the fraction of unsuitable patches, or the fraction of habitat loss). The non-trivial equilibrium of Eq. (1), $p^{*}=h-e / c$, is globally stable if $h>e / c$. Therefore, the minimum fraction of suitable patches necessary for metapopulation persistence is defined by the ratio of the extinction rate to the colonization rate, and the fraction of empty suitable patches remains constant (i.e. $h-p^{*}=e / c$ ) unless the metapopulation becomes extinct, known as the Levins rule [20].

In the following, we assume that the suitable patches are destructed at a certain rate $(d)$ due to human activities or natural causes (e.g. wild fires and hurricanes). The unsuitable patches can be restored at a certain rate $(\mu)$ through external habitat restoration by the activities of other organisms (e.g. their metabolism), abiotic causes (e.g. self recovering) and human endeavor (e.g. conservation effort). Moreover, the unsuitable patches can also be restored by the organism itself at a certain rate $(\lambda)$, i.e. organism-induced internal habitat restoration. Accordingly, we have the following model by extending Eq. (1) (see Appendix A for a derivation from the Markov process):

$$
\begin{aligned}
& \frac{d p}{d t}=c p(h-p)-(e+d) p \\
& \frac{d h}{d t}=(\lambda p+\mu)(1-h)-d h
\end{aligned}
$$

Notice that all parameters $(c, e, d, \lambda$ and $\mu$ ) are non-negative, and that $p \leqslant h$. We define $\alpha=\lambda / d$ and $\beta=\mu / d$ in the following analysis as the intensities of the internal and external processes of habitat restoration, relative to habitat destruction (at a rate of $d$ ). Let $\delta=(e+d) / c$ indicate the decreasing rate of the fraction of occupied patches (including the natural extinction of local populations and the patch loss due to habitat destruction) relative to the colonization rate (see Table 1 for details). Eq. (2) implies that the habitat loss is completely random, meaning that (i) all suitable patches including occupied and empty patches may be destroyed at the same rate, and (ii) when an occupied patch is destroyed the local population within the patch goes to extinction simultaneously.

\section{Analysis}

Eq. (2) has a unique boundary equilibrium $(0, \beta /(\beta+1))$. Obviously, this boundary equilibrium corresponds to the extinction of metapopulation. We have the following two theorems for its stability.

Theorem 1. The boundary equilibrium $(0, \beta /(\beta+1))$ is locally asymptotically stable if $\delta>\beta /(\beta+1)$, and it is unstable if $\delta<\beta /(\beta+1)$.

Proof. The Jacobian matrix of Eq. (2) at the boundary equilibrium $(0, \beta /(\beta+1))$ is:

$J_{\text {boundary }}=\left(\begin{array}{cc}\frac{c \beta}{\beta+1}-(e+d) & 0 \\ \frac{\lambda}{\beta+1} & -(\mu+d)\end{array}\right)$,

with two eigenvalues $c \beta /(\beta+1)-(e+d)$ and $-(\mu+d)$. Thus, the boundary equilibrium $(0, \beta /(\beta+1))$ is unstable if $\delta<\beta /(\beta+1)$ (Fig. 1A) and locally asymptotically stable if $\delta>\beta /(\beta+1)$ (Fig. 1DF).
Table 1

Parameters used in the patch occupancy model with habitat restoration.

\begin{tabular}{ll}
\hline Parameter & Description \\
\hline$c$ & Colonization rate to empty suitable patches \\
$e$ & Extinction rate of local populations \\
$d$ & Habitat destruction rate \\
$\lambda$ & Internal habitat restoration rate \\
$\mu$ & External habitat restoration rate \\
$\alpha=\lambda / d$ & Relative intensity of internal restoration to habitat destruction \\
$\beta=\mu / d$ & Relative intensity of external restoration to habitat destruction \\
$\delta=(e+d) / c$ & Relative decrease rate of occupied patch fraction to colonization \\
& rate \\
\hline
\end{tabular}

Theorem 2. If $\delta=\beta /(\beta+1)$, then the boundary equilibrium $(0, \beta)$ $(\beta+1))$ is locally asymptotically stable if and only if $\alpha \leqslant(\beta+1)^{2}$. Here the stability refers only to the local attraction of positive trajectories (see Appendix B for the proof; Fig. 1B, C).

There are at most two interior equilibriums for the Eq. (2) (see Appendix $C$ for details and Table 2 for a summary). For convenience, let $\left(p^{*}, h^{*}\right)$ denotes the interior equilibrium of Eq. (2). On the $p$ - $h$ phase plane, the zero isocline for $d p / d t=0$, denoted by $L_{1}$, is determined by the equation $c(h-p)-(e+d)=0$, and the zero isocline for $d h / d t=0$, denoted by $L_{2}$, is determined by the equation $(\lambda p+\mu)(1-h)-d h=0$. It is easy to see that the slope of $L_{1}$ is one, and the slope of $L_{2}$ at interior equilibrium $\left(p^{*}, h^{*}\right)$ is $\alpha\left(1-h^{*}\right)$ / $\left(\alpha p^{*}+\beta+1\right)$. Subsequently, for the stability of $\left(p^{*}, h^{*}\right)$, we have the following theorem.

Theorem 3. An interior equilibrium of Eq. (2), $\left(p^{*}, h^{*}\right)$, is locally asymptotically stable if and only if the slope of $L_{2}$ at the interior equilibrium satisfies $\alpha\left(1-h^{*}\right) /\left(\alpha p^{*}+\beta+1\right)<1$.

Proof. The Jacobian matrix of Eq. (2) at $\left(p^{*}, h^{*}\right)$ is

$J_{\left(p^{*}, h^{*}\right)}=\left(\begin{array}{cc}-c p^{*} & c p^{*} \\ \lambda\left(1-h^{*}\right) & -\left(\lambda p^{*}+\mu+d\right)\end{array}\right)$.

Notice that the eigenvalues of the matrix $J_{\left(p^{*}, h^{*}\right)}$ are

$-\frac{1}{2}\left(\left(c p^{*}+\omega^{*}\right) \pm \sqrt{\left(c p^{*}+\omega^{*}\right)^{2}-4 c p^{*} \omega^{*}\left(1-\frac{\alpha\left(1-h^{*}\right)}{\alpha p^{*}+\beta+1}\right)}\right)$,

where $\omega^{*}=\lambda p^{*}+\mu+d$. Thus, $\left(p^{*}, h^{*}\right)$ is locally asymptotically stable if $\alpha\left(1-h^{*}\right) /\left(\alpha p^{*}+\beta+1\right)<1$ (Fig. 1A, B, D), and unstable if $\alpha\left(1-h^{*}\right) /\left(\alpha p^{*}+\beta+1\right)>1$ (Fig. 1D). For $\alpha\left(1-h^{*}\right) /\left(\alpha p^{*}+\beta+1\right)=1$, one of the two eigenvalues is zero, and the other is negative. Eq. (2) can be equivalently rewritten as (similarly to the proof in Theorem 2, see Appendix B)

$$
\begin{aligned}
& \frac{d \tilde{p}}{d t}=\frac{\lambda c p^{*}}{\left(c p^{*}+\omega^{*}\right)^{2}} \tilde{p}^{2}+\xi(\tilde{p}, \tilde{h}) \\
& \frac{d \tilde{h}}{d t}=-\left(c p^{*}+\omega^{*}\right) \tilde{h}+\zeta(\tilde{p}, \tilde{h}),
\end{aligned}
$$

where $\tilde{p}=-\omega^{*} p-c p^{*} h+\omega^{*} p^{*}+c h^{*} p^{*}$ and $\tilde{h}=\omega^{*} p-\omega^{*} h-\omega^{*} p^{*}$ $+\omega^{*} h^{*}$, and the function $\xi(\tilde{p}, \tilde{h})$ and $\zeta(\tilde{p}, \tilde{h})$ are polynomials with respect to $\tilde{p}$ and $\tilde{h}$ (the first-degree terms are excluded). Therefore, in term of the center manifold theory [21], there exist a local center manifold $\tilde{h}=\varphi(\tilde{p})$ with $\varphi(0)=0$ and $d \varphi(0) / d t=0$, which can be obtained by solving the equation

$\frac{d \varphi(\tilde{p})}{d \tilde{p}}\left[\frac{\lambda c p^{*}}{\left(c p^{*}+\omega^{*}\right)^{2}} \tilde{p}^{2}+\xi(\tilde{p}, \varphi(\tilde{p}))\right]=-\left(c p^{*}+\omega^{*}\right) \varphi(\tilde{p})+\zeta(\tilde{p}, \varphi(\tilde{p}))$.

Thus, the stability of system (2) is determined by the reduced system 

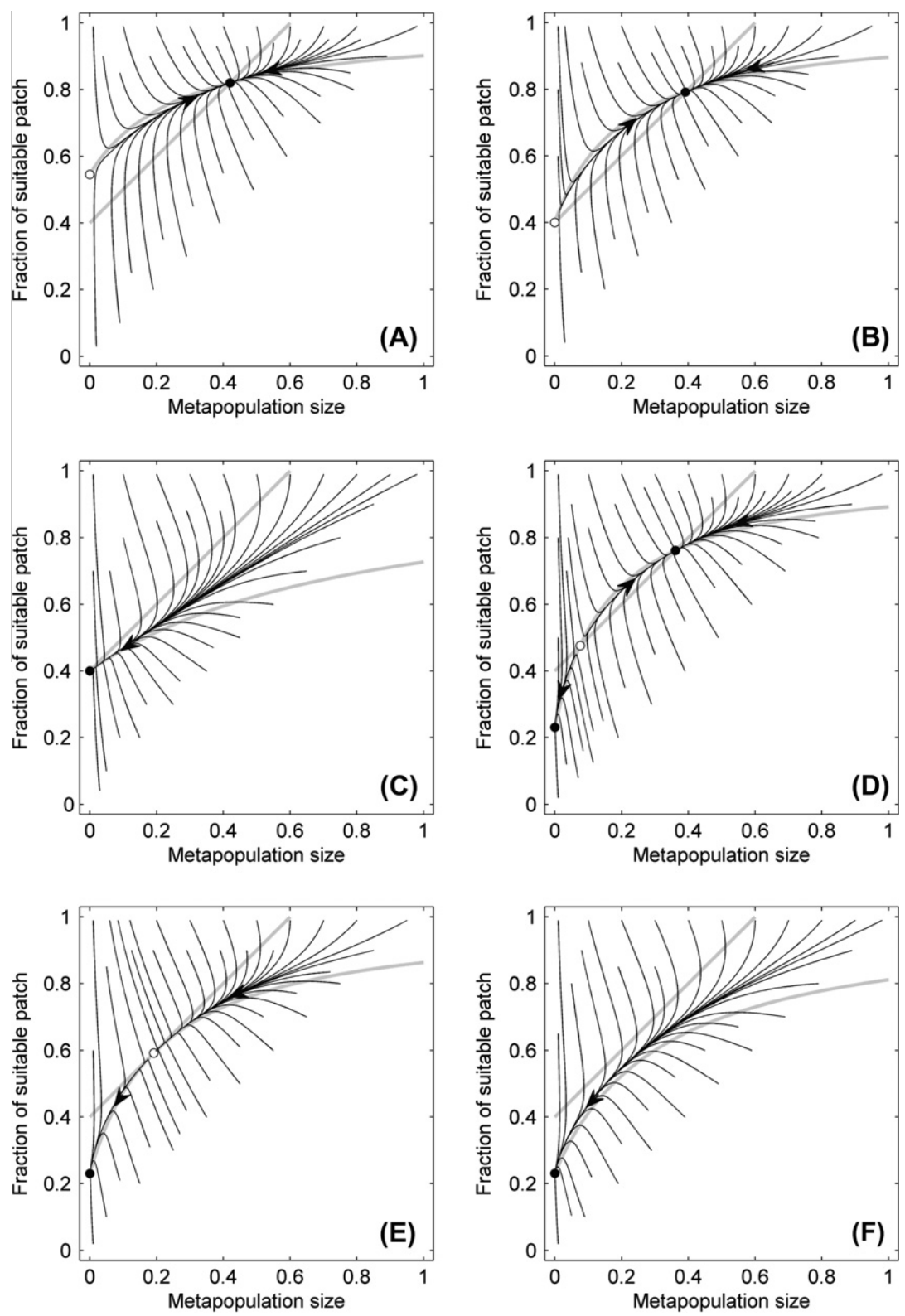

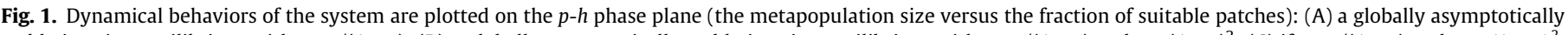

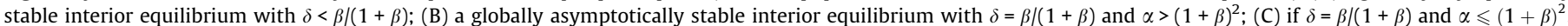

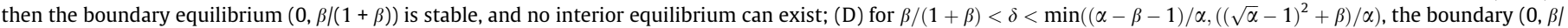

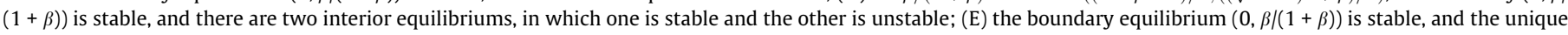

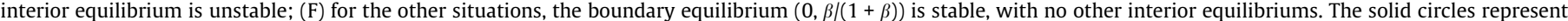

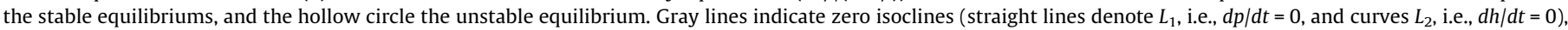

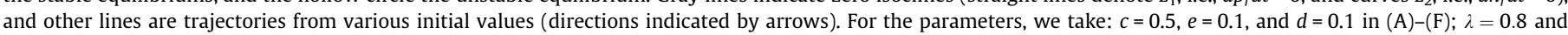
$\mu=0.12$ in (A); $\lambda=0.8$ and $\mu=2 / 30$ in (B); $\lambda=0.2$ and $\mu=2 / 30$ in (C), $\lambda=0.8$ and $\mu=0.03$ in (D), $\lambda=0.6$ and $\mu=0.0299$ in (E); $\lambda=0.4$ and $\mu=0.03$ in (F).

Table 2

The equilibrium and stability of Eq. (2) and the metapopulation persistence.

\begin{tabular}{|c|c|c|c|}
\hline Parameter condition & Boundary equilibrium & Interior equilibrium & Metapopulation persistence \\
\hline$\delta<\frac{\beta}{\beta+1}$ & Unstable & (One) $)^{*}$ Stable & Persistence (Fig. 1A) \\
\hline$\delta=\frac{\beta}{\beta+1}$ and $\alpha>(\beta+1)^{2}$ & Unstable & (One) Stable & Persistence (Fig. 1B) \\
\hline$\frac{\beta}{\beta+1}<\delta<\min \left(\frac{\alpha-\beta-1}{\alpha}, \frac{(\sqrt{\alpha}-1)^{2}+\beta}{\alpha}\right)$ & Stable & (Two) Unstable stable & Persistence depends on the initial state (Fig. 1D) \\
\hline \multirow{2}{*}{$\begin{array}{l}\delta=\frac{(\sqrt{\alpha}-1)^{2}+\beta}{\alpha} \text { and } \alpha>(\beta+1)^{2} \\
\text { Others }\end{array}$} & Stable & (One) unstable & Extinction (Fig. 1E) \\
\hline & Stable & (None) & Extinction (Fig. 1C\&F) \\
\hline
\end{tabular}

* Parentheses indicate the number of interior equilibrium. 
$\frac{d \tilde{p}}{d t}=\frac{\lambda c p^{*}}{\left(c p^{*}+\omega^{*}\right)^{2}} \tilde{p}^{2}+o\left(\tilde{p}^{2}\right)$

i.e., $\left(p^{*}, h^{*}\right)$ is unstable if $\alpha\left(1-h^{*}\right) /\left(\alpha p^{*}+\beta+1\right)=1$ (Fig. 1E).

Subsequently, a straightforward question here is whether the existence of periodic solution is possible. The following theorem shows that no periodic solution exists in Eq. (2).

Theorem 4. For Eq. (2), the existence of periodic solution is impossible.

Proof. The Dulac function is defined as $\psi(p, h)=1 / p$, and notice that

$\frac{\partial(\psi P)}{\partial p}+\frac{\partial(\psi Q)}{\partial h}=-\left(c+\frac{\lambda p+\mu+d}{p}\right)<0$

for all positive $p$ and $h$, where $P(p, h)=d p / d t$ and $Q(p, h)=d h / d t$. Thus, from The Bendixson-Dulac theorem, no periodic solution can exist in Eq. (2).

Theorem 4 implies that, if there is a unique interior equilibrium and it is also locally asymptotically stable, then it must be globally asymptotically stable, because, in this situation, the unique boundary equilibrium is unstable (see Table 2 and Fig. 1A, B).

According to the above analysis, the dynamical properties of Eq. (2) can be summarized as: (i) if the boundary equilibrium $(0, \beta)$ $(\beta+1))$ is unstable, then there exists a unique interior equilibrium that is globally asymptotically stable (Fig. 1A, B); (ii) if the boundary equilibrium $(0, \beta /(\beta+1))$ is stable, then there are at most two interior equilibriums; (iii) no periodic solution can exist in the system. The situation (ii) further includes three scenarios: (a) the situation with no interior equilibrium is possible (Fig. 1C, F); (b) if there exists only one interior equilibrium, then it must be unstable saddle point (Fig. 1E); (c) if there are two interior equilibriums, the one is locally asymptotically stable but the other unstable (Fig. 1D). All of these results are summarized in Table 2 . The case with two interior equilibriums indicates a bi-stable system, and thereby the fate of metapopulation depends on its initial size, i.e., a threshold phenomenon.
The relationship between the equilibrium of metapopulation size and the internal restoration intensity $\alpha(=\lambda / d)$ is plotted in Fig. 2. If the value of $\beta(=\mu / d$; the external restoration intensity) is small $(\beta<\delta /(1-\delta))$, the bi-stability will occur when the internal restoration intensity $(\alpha)$ is high (Fig. $2 \mathrm{~A}, \mathrm{~B}$ ). No bi-stability exists if $\beta \geqslant \delta /(1-\delta)$ (Fig. 2C, D). Similarly, the relationship between the equilibrium of metapopulation size and the external restoration intensity $\beta$ is plotted in Fig. 3. If $\alpha$ equals zero (or small), the system of Eq. (2) is essentially similar to the Levins model, with $\beta /(\beta+1)$ analogous to $h$ as in Eq. (1) (Fig. 3A, B). If $\alpha$ is large (determined by Eq. (C6) in Appenix C), the bi-stability will occur when $\beta$ is moderate (Fig. 3C, D). Therefore, strong habitat restoration induced by the organism itself plays a crucial role to trigger the bistability, whereas the intensity of external restoration is a key to eliminate the bi-stability.

\section{Discussion}

In this paper, following Levins' [3,4] patch occupancy model of metapopulations, we developed a theoretical model that describes not only metapopulation dynamics but also habitat dynamics induced by two processes of habitat destruction and restoration. The internal habitat restoration induced by the organism itself is separated from the external restoration by other biotic and abiotic causes. The positive feedback relationship between the habitat and metapopulation dynamics has been revealed by a full stability analysis of the system. The outcome implies that the organismenvironment interaction can affect the persistence of metapopulation significantly to a certain degree (reflected by the relative strength of habitat restoration, $\alpha$ and $\beta$ ). If the habitat destruction is inevitable, then the habitat restoration possibly driven by the organism itself and other conservation efforts are crucial for the metapopulation persistence (see Table 2 for details).

The evaluation of the persistence of a species inhabiting fragmented habitat is important in ecology [22]. Multiple factors, such as habitat destruction [23], environmental stochasticity [24], demographic stochasticity [5,25], dynamical complexity [26], behavior evolution [27,28] and rapid evolution [29], have been identified to be able to affect the persistence of metapopulations. In addition, our results suggested that the internal habitat restora-
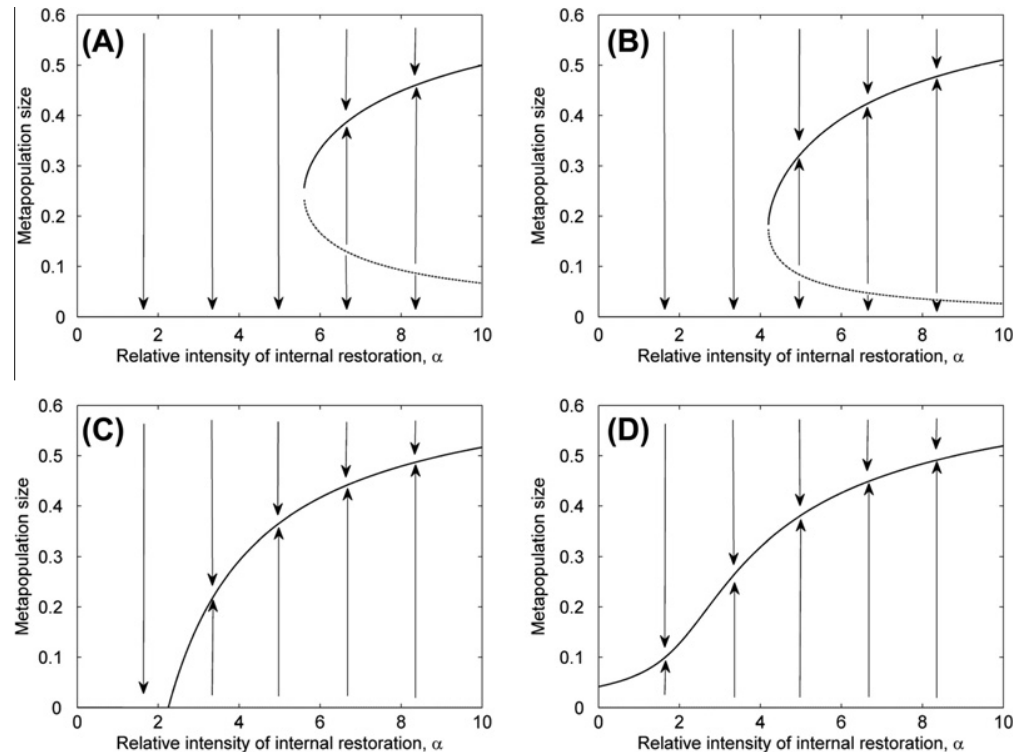

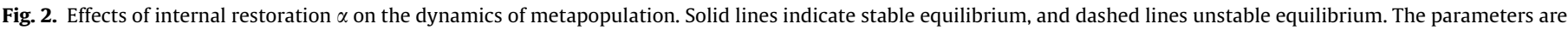
taken as $\delta=1 / 3$, and $\beta=0,0.3,0.5$ and 0.6 in (A)-(D), respectively. 

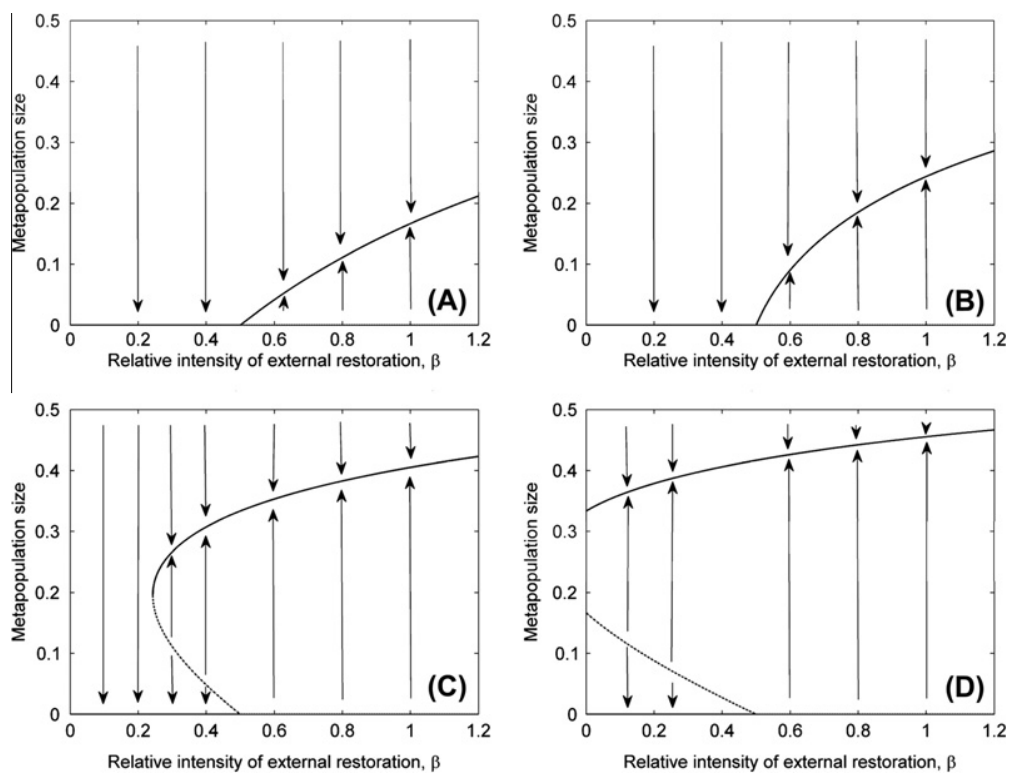

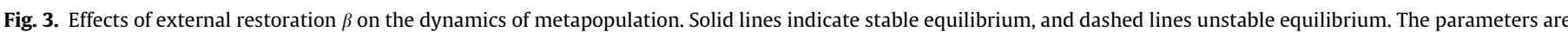
taken as $\delta=1 / 3$, and $\alpha=0,1.5,4.5$ and 6 in (A)-(D), respectively.

tion by the organisms can also promote the persistence of their metapopulations and thus increases the metapopulation size. However, whether a biological system can restore to its initial status depends not only on its capacity of habitat restoration but also on the level of habitat destruction. A highly destructed habitat could have potentially altered the system equilibrium from positive to extinction, making small-scale restoration in vain.

Internal restoration induced by the organism itself is the trigger of the bi-stability (or the threshold phenomenon) of the system, whereas the external restoration can eliminate this bi-stability (Fig. 3). This threshold phenomenon could arise from the density-dependent negative growth rate at low metapopulation size, indicating a sufficient number of suitable patches or metapopulation size for persistence. Other mechanisms that can cause such negative growth rate and threshold phenomenon have been identified, such as the Allee effect [30,31] and the rescue effect [2]. The organism-environment feedback is a crucial interaction in ecosystems $[10,32]$, yet often neglected in biological conservation. This positive feedback (i.e. the capacity of habitat restoration) can strongly affect the distribution and the abundance of species [32], the consequence of competition [16], the structure and functioning of ecosystem [33-35], evolutionary directions [36], the maintenance of polymorphism $[17,18]$, and population dynamics [37-39]. Our study here suggests that organism-induced habitat restoration can profoundly affect the persistence of metapopulations, change stable systems into bi-stable ones, cause the threshold phenomenon, and thus should merit further investigations.

\section{Acknowledgements}

This research was supported by the National Basic Research Program (973 Program) (No. 2007CB109107), Gansu Natural Science Fund (No. 1010RJZA127), the NRF Incentive Research Programme, and the DST-NRF Centre of Excellence for Invasion Biology at Stellenbosch University.

\section{Appendix A. Derivation of Eq. (2) from the Markov stochastic process}

Consider a total number of $z$ patches in the landscape, including a number of $x$ occupied patches and a number of $y$ suitable patches
$(0 \leqslant x \leqslant y \leqslant z)$; we can thus define a continuous-time Markov process with the following non-zero transition rates:

$$
\begin{array}{cc}
\text { Event } & \text { Rate } \\
(x, y) \rightarrow(x+1, y) & c^{\prime} m x(y-x) / z \\
(x, y) \rightarrow(x-1, y) & \text { ex } \\
(x, y) \rightarrow(x, y+1) & (\mu+r m x / z)(z-y) \\
(x, y) \rightarrow(x, y-1) & d(y-x) \\
(x, y) \rightarrow(x-1, y-1) & d x
\end{array}
$$

where $m$ is the number of emigrants from each occupied patch, $c^{\prime}$ the colonization coefficient and $e$ the extinction rate. It describes the following Markov process: emigrants randomly land into patches regardless of the patch status, and thus each gets $m x / z$ immigrants; for an empty suitable patch, the probability of successful colonization is $c^{\prime} m x / z$; for an occupied patch, the patch status remains; for an unsuitable patch, the immigrants contribute to restoring the patch by organism-induced way, and the restoration rate of an unsuitable patch is thus $\mu+r m x / z$. The above Markov process can also be approximated for large $z$ using the following twodimension ordinary differential equations [40]:

$$
\begin{aligned}
& \frac{d x}{d t}=c^{\prime} \frac{m x}{z}(y-x)-e x-d x \\
& \frac{d y}{d t}=\left(r \frac{m x}{z}+\mu\right)(z-y)-d(y-x)-d x .
\end{aligned}
$$

If we let $c=c^{\prime} m, \lambda=r m, p=x / z$ (the fraction of occupied patches) and $h=y / z$ (the fraction of suitable patches), Eq. (A2) becomes Eq (2) in the main text.

\section{Appendix B. Proof of Theorem 2}

If $\delta=\beta /(\beta+1)$, then one of the eigenvalues of the Jacobian matrix $J_{\text {boundary }}$ must be zero. Let $\tilde{p}=p$ and $\tilde{h}=h-\beta \gamma-\alpha \gamma^{2}$, where $\gamma=1 /(\beta+1)$, then Eq. (2) can be equivalently expressed as:

$$
\begin{aligned}
& \frac{d \tilde{p}}{d t}=c\left(\alpha \gamma^{2}-1\right) \tilde{p}^{2}+c \tilde{p} \tilde{h} \\
& \frac{d \tilde{h}}{d t}=-(\mu+d) \tilde{h}-\pi(\tilde{p}, \tilde{h}),
\end{aligned}
$$

where $\pi(\tilde{p}, \tilde{h})=\left(\lambda+c \alpha \gamma^{2}\right) \tilde{p} \tilde{h}+\left[\alpha \gamma^{2}(\lambda+c)-c \alpha^{2} \gamma^{4}\right] \tilde{p}^{2}$. From the center manifold theory [21], there exists a local center manifold 
$\tilde{h}=\varphi(\tilde{p})$ with $\varphi(0)=0$ and $d \varphi(0) / d \tilde{p}=0$. Notice that $\tilde{h}=\varphi(\tilde{p})$ satisfies

$\frac{d \varphi(\tilde{p})}{d \tilde{p}}\left[c\left(\alpha \gamma^{2}-1\right) \tilde{p}^{2}+c \tilde{p} \varphi(\tilde{p})\right]=-(\mu+d) \varphi(\tilde{p})-\pi(\tilde{p}, \varphi(\tilde{p}))$,

and that $\varphi(\tilde{p})$ can be solvable. Thus, the stability of $(0, \beta /(\beta+1))$ is completely determined by the reduced system:

$\frac{d \tilde{p}}{d t}=c\left(\alpha \gamma^{2}-1\right) \tilde{p}^{2}+c \tilde{p} \varphi(\tilde{p})$.

Since $c \tilde{p} \varphi(\tilde{p})=o\left(\tilde{p}^{2}\right)$, the boundary equilibrium $(0, \beta /(\beta+1))$ is a saddle point if $\alpha \gamma^{2} \neq 1$. Specifically, $(0, \beta /(\beta+1))$ is unstable for positive trajectories if $\alpha \gamma^{2}>1$ (Fig. 1B), and it is a local attractor for positive trajectories if $\alpha \gamma^{2}<1$ (Fig. 1C). For the case with $\alpha \gamma^{2}=1$, the solution of Eq. (B3) can be expressed as $\varphi(\tilde{p})=-\alpha \gamma \tilde{p}^{2}+o\left(\tilde{p}^{2}\right)$, and Eq. (B3) can be rewritten as

$\frac{d \tilde{p}}{d t}=-c \alpha \gamma \tilde{p}^{3}+o\left(\tilde{p}^{3}\right)$.

Thus, $(0, \beta /(\beta+1))$ is locally asymptotically stable if $\alpha \gamma^{2}=1$.

\section{Appendix C. Interior equilibrium of Eq. (2)}

The interior equilibrium of Eq. (2) must be the solution of the following equations

$$
\begin{aligned}
& c(h-p)-(e+d)=0 \\
& (\lambda p+\mu)(1-h)-d h=0,
\end{aligned}
$$

Notice that these equations can be equivalently expressed as

$\alpha p^{2}-[\alpha(1-\delta)-\beta-1] p+(\beta+1) \delta-\beta=0$.

Thus, Eq. (2) has at most two interior equilibriums, and the necessary condition for the existence of an interior equilibrium is $(\alpha(1-\delta)+\beta+1)^{2}-4 \alpha \geqslant 0$. In order to determine whether there exists an interior equilibrium, let

$f(p)=\alpha p^{2}-(\alpha(1-\delta)-\beta-1) p+(1+\beta) \delta-\beta$.

It is straightforward to see that

$f(0)=(\beta+1) \delta-\beta$

$f(1)=(\alpha+\beta-1) \delta+1$,

and that the minimum point of $f(p)$ is

$\hat{p}=\frac{\alpha(1-\delta)-\beta-1}{2 \alpha}$.

Since a necessary condition for the existence of a positive equilibrium is $e+d<c$ (i.e., $\delta<1)[41,42]$, we have $f(1)>0$. This implies that (i) if $f(0)<0$, i.e. $\delta<\beta /(\beta+1)$, then there could be only one interior equilibrium (Fig. $1 \mathrm{~A}$ ); (ii) under the condition $[\alpha(1-\delta)+\beta+1]^{2}-4 \alpha \geqslant 0$, if $f(0)>0$ and $0<\hat{p}<1$, i.e.,

$\frac{\beta}{\beta+1}<\delta<\min \left(\frac{\alpha-\beta-1}{\alpha}, \frac{(\sqrt{\alpha}-1)^{2}+\beta}{\alpha}\right)$,

two interior equilibriums can occur (Fig. 1D); (iii) for $f(0)=0$, i.e., $\delta=\beta /(\beta+1)$, only one interior equilibrium occurs when $\alpha>(\beta+1)^{2}$ (Fig. 1B), and no interior equilibrium exists when $\alpha \leqslant(\beta+1)^{2} \quad$ (Fig. 1C); (iv) for $[\alpha(1-\delta)+\beta+1]^{2}-4 \alpha=0$, i.e. $\delta=\left((\sqrt{\alpha}-1)^{2}+\beta\right) / \alpha$, there is only one interior equilibrium when $\alpha>(\beta+1)^{2}$ (Fig. 1E); and (v) for other situations, there is no any interior equilibrium (Fig. 1F). These results were summarized in Table 2.

\section{References}

[1] D.S. Wilcove, D. Rothstein, D. Jason, A. Phillips, E. Losos, Quantifying threats to imperiled species in the United States, Bioscience 48 (1998) 607-615.

[2] I. Hanski, Metapopulation Ecology, Oxford University Press, Oxford, 1999.

[3] R. Levins, Some demographic and genetic consequence of environmental heterogeneity for biological control, Bull. Entomol. Soc. Am. 15 (1969) 237.

[4] R. Levins, Extinction, Lect. Notes Math. 2 (1970) 75.

[5] R. Lande, Extinction thresholds in demographic models of territorial populations, Am. Nat. 130 (1987) 624.

[6] R.M. May, The role of ecological theory in planning reintroduction of endangered species, Symp. Zool. Soc. London 62 (1991) 145.

[7] S. Nee, R.M. May, Dynamics of metapopulations: habitat destruction and competitive coexistence, J. Ani. Ecol. 61 (1992) 37.

[8] J.H. Lawton, S. Nee, A.J. Letcher, P.H. Harvey, Animal distributions: pattern and process, in: P.J. Edwards, R.M. May (Eds.), Large-scale Ecology and Conservation Biology, Blackwell Scientific Press, Oxford, 1994, pp. 41-58.

[9] S. Nee, How populations persist, Nature 367 (1994) 123.

[10] C.G. Jones, J.H. Lawton, M. Shachak, Organisms as ecosystem engineers, Oikos 69 (1994) 373.

[11] A. Hastings, J.E. Byers, J.A. Crooks, K. Cuddington, C.G. Jones, J.G. Lambrinos, T.S. Talley, W.G. Wilson, Ecosystem engineering in space and time, Ecol. Lett. 10 (2007) 153.

[12] J.E. Byers, K. Cuddington, C.G. Jones, T.S. Talley, A. Hastings, J.G. Lambrinos, J.A. Crooks, W. Wilson, Using ecosystem engineers to restore ecological systems, Trends Ecol. Evol. 21 (9) (2006) 493.

[13] D.M. Mager, C. Hui, A first record of biological soil crusts in the Cape Floristic Region, South African J. Sci. 108 (7/8) (2012) p. 4. Art. \#1013.

[14] P.L. Zarnetske, S.D. Hacker, E.W. Seabloom, P. Ruggiero, J.R. Killian, T.B. Maddux, D. Cox, Biophysical feedback mediates effects of invasive grasses on coastal dune shape, Ecology 93 (6) (2012) 1439.

[15] D.X. Yue, C. Hui, Z.Z. Li, Niche construction for desert plants in individual and population scales: theoretical analysis and evidences from saksaul (Haloxylon ammodendron) forests, Israel J. Plant Sci. 52 (2004) 235.

[16] C. Hui, Z.Z. Li, D.X. Yue, Metapopulation dynamics and distribution, and environmental heterogeneity induced by niche construction, Ecol. Model. 177 (2004) 107.

[17] C. Hui, D.X. Yue, Niche construction and polymorphism maintenance in metapopulation, Ecol. Res. 20 (2005) 115.

[18] X.Z. Han, Z.Z. Li, C. Hui, F. Zhang, Polymorphism maintenance in a spatially structured population: a two-locus genetic model of niche construction, Ecol. Model. 192 (2006) 160

[19] X.Z. Han, C. Hui, Y.Y. Zhang, Effects of time-lagged niche construction on metapopulation dynamics and environmental heterogeneity, Appl. Math. Comput. 215 (2009) 449.

[20] I. Hanski, A. Moilanen, M. Gyllenberg, Minimum viable metapopulation size, Am. Nat. 147 (1996) 527.

[21] J. Carr, Applications of Center Manifold Theory, Springer, New York, 1981.

[22] R. Casagrandi, M. Gatto, A persistence criterion for metapopulations, Theor Popul. Biol. 61 (2002) 115.

[23] M. Gyllenberg, I. Hanski, Habitat deterioration, habitat destruction, and metapopulation persistence in a heterogenous landscape, Theor. Popul. Biol. 52 (1997) 198.

[24] O.L. Petchey, A. Gonzalez, H.B. Wilson, Effects on population persistence: the interaction between environmental noise colour, intraspecific competition and space, Proc. Biol. Sci. 264 (1997) 1841.

[25] P.L. Chesson, Persistence of a Markovian population in a patchy environment, Z. Wahrscheinlichkeitstheor. 66 (1984) 97.

[26] C. Hui, Z.Z. Li, Dynamical complexity and metapopulation persistence, Ecol. Model. 164 (2003) 201.

[27] F. Zhang, C. Hui, X.Z. Han, Z.Z. Li, Evolution of cooperation in patchy habitat under patch decay and isolation, Ecol. Res. 20 (2005) 461.

[28] C. Hui, F. Zhang, X.Z. Han, Z.Z. Li, Cooperation evolution and self-regulation dynamics in metapopulation: stage-equilibrium hypothesis, Ecol. Model. 184 (2005) 397.

[29] M.A. Patten, D.H. Wolfe, E. Shochat, S.K. Sherrod, Habitat fragmentation, rapid evolution and population persistence, Evol. Ecol. Res. 7 (2005) 235.

[30] W.C. Allee, The Social Life of Animals, Heinemann, London, 1938.

[31] C.M. Taylor, A. Hastings, Allee effects in biological invasions, Ecol. Lett. 8 (2005) 895.

[32] J.P. Wright, C.G. Jones, The concept of organisms as ecosystem engineers ten years on: progress, limitations, and challenges, Bioscience 56 (2006) 203.

[33] F.S. Chapin, B.H. Walker, R.J. Hobbs, D.U. Hooper, J.H. Lawton, O.E. Sala, D. Tilman, Biotic control over the functioning of ecosystems, Science 277 (1997) 500.

[34] E.I. Badano, L.A. Cavieres, Impacts of ecosystem engineers on community attributes: effects of cushion plants at different elevations of the Chilean Andes, Diversity Distrib. 12 (2006) 388.

[35] E.I. Badano, C.G. Jones, L.A. Cavieres, J.P. Wright, Assessing impacts of ecosystem engineers on community organization: a general approach illustrated by effects of a high-Andean cushion plant, Oikos 115 (2006) 365.

[36] K.N. Laland, F.J. Odling-Smee, M.W. Feldman, Evolutionary consequences of niche construction and their implications for ecology, Proc. Nat. Acad. Sci. USA 96 (1999) 10242. 
[37] W.S.C. Gurney, J.H. Lawton, The population dynamics of ecosystem engineers, Oikos 76 (1996) 273.

[38] K. Cuddington, W.G. Wilson, A. Hastings, Ecosystem engineers: feedback and population dynamics, Am. Nat. 173 (2009) 488.

[39] J.P. Wright, W.S.C. Gurney, C.G. Jones, Patch dynamics in a landscape modified by ecosystem engineers, Oikos 105 (2004) 336.
[40] T. Kurtz, Solution of ordinary differential equations as limits of pure jump Markov process, J. Appl. Probab. 7 (1970) 49.

[41] I. Hanski, Metapopulation dynamics, Nature 396 (1998) 41.

[42] F. Zhang, Z.Z. Li, C. Hui, Spatiotemporal dynamics and distribution patterns of cyclic competition in metapopulation, Ecol. Model. 193 (2006) 721. 